\title{
ABCDEFG IS - The Principle of Constructive Feedback
}

\author{
Bhattarai $M D^{1}$ \\ ${ }^{1}$ Medical Education Unit, National Academy of Medical Sciences, Bir hospital , Kathmandu.
}

\begin{abstract}
Feedback is an integral part of any learning experience. Constructive feedback is a powerful instrument and facilitates the learner's professional and personal development. "ABCDEFG IS", a mnemonic for the principles of constructive feedback, stands for Amount of the information, Benefit of the trainees, Change behaviour, Descriptive language, Environment, Focused, Group check, Interpretation check, and Sharing information. The eight important steps of feedback are: Ensure prior information, Collect data, Make appropriate meeting arrangement, Begin by encouraging self assessment by the trainee, Highlight areas where the trainee is doing well, Give feedback, Handle reaction maintaining the dignity and Plan actions. Communication and reflection also share many of the principles and steps of constructive feedback and giving regular feedback, thus, helps to improve communication and reflection. The feedback provider would be able to provide genuine feedback by following the appropriate steps and principles of constructive feedback and realize how important and rewarding its role is in teaching learning activities.
\end{abstract}

Key Words: Constructive feedback, Feedback, Feedback steps.

\section{INTRODUCTION}

Feedback is an essential part of learning. Giving constructive feedback facilitates learning and forms an important role of a teacher. It is of little use if the information obtained does not get back where it does the most good - back to the trainees. As professionals we are also responsible for ensuring we receive feedback on our own performance. Feedback gives insight and stimulates reflection. It helps motivation and may challenge attitudes and values. The aims of feedback are to encourage the changes in behaviour that are required for trainees to reach and maintain appropriate professional standards as well as to enhance the personal and professional self esteem of learners. Feedback may help change behaviour in a variety of ways: ${ }^{1}$
- By offering strategies to help a learner improve

- By enhancing the learner's professional and personal self esteem

- By reinforcing the learner's strength and

- By identifying areas of professional or clinical weakness. Feedback is a powerful instrument, which, if used wisely, can facilitate the trainee's professional and personal development. If the faculty does not provide feedback to the students, it will be injustice to the student, who is not doing well, and also to the student, who is doing well. By avoiding an issue, or ignoring poor performance, we are discriminating against those trainees who work hard to do well. We may be prejudicing the welfare of patients as well as the integrity of our profession. If trainees do not receive feedback, they will generate their

\section{Correspondence: \\ Dr. Madhur Dev Bhattarai \\ Medical Education Unit, NAMS \\ Bir hospital, Kathmandu, Nepal. \\ Email: mdb@ntc.net.np}


own system of feedback, largely based on unintended cues we provide e.g. the fact that we haven't criticized means that all was fine. ${ }^{1}$ It is important not to offer meaningless or dishonest feedback e.g. "that was OK" when it was poorly done.

\section{BARRIERS}

It is now getting widely accepted that learners need effective feedback on their performances in order to develop and improve their skills. However there are many barriers to providing effective feedback. ${ }^{2}$ Although students are eager for feedback, they may feel uncomfortable in interacting with faculty for seeking feedback. Also, since student's self-assessment skills are not well developed yet, they may not know what questions to ask. A large number of faculty members, who have had no formal concept and experience in methods of giving effective feedback, feel uncomfortable in giving specific feedback to students. Faculty often lack confidence in their observations, have fears of 'hurting the student's feeling', or simply do not know how to translate their observations into specific and constructive feedback. ${ }^{2}$ Consequently, feedback is often very general and not helpful to students. In order to avoid offering students potentially difficult feedback, tutors may award everybody the highest grade. ${ }^{1}$ Even when faculty think they have given detailed feedback, students often consider the process to be inadequate. ${ }^{3}$ Moreover, feedback in medical education tended to emphasize the learners' omissions and failures while omitting any supportive or constructive advice on how to change. ${ }^{4}$ Learners felt that feedback was a destructive experience and one to be avoided. Unfortunately, students still report instances of teaching by humiliation. ${ }^{5}$

ABCDEFG IS - The Principle of Constructive Feedback There are certain principles of constructive feedback, which can be adapted to a variety of educational situations. ${ }^{1,6,7}$ The principles of constructive feedback are by no means new. They have been available for over a quarter of a century, yet have not infiltrated medical education to an appreciable extent. To make it easier to remember, the author has described them below with the mnemonic of "ABCDEF IS", which stands for Amount of the information, Benefit of the trainees, Change behaviour, Descriptive language, Environment, Focused, Group check, Interpretation check, and Sharing information.

\section{A. Amount of the information}

The amount of information in the feedback may need to be limited. Too much of information may overload the trainees. The feedback should be limited to the amount of information that the recipient can use rather than the amount faculty members would like to give.

\section{B. Benefit of the trainees}

Though it is obvious, it is strange that many times while giving feedback the faculty members tend to forget that feedback should be offered for the learner's benefit. Feedback may be provided with well intention. But patronizing, mocking superior comments are provided for the benefits of the observer rather than helping and encouraging the learner. Feedback should be given that serves the needs of the learner, rather than the needs of the provider. ${ }^{6}$ It should not be simply a method of providing 'release' to the giver. The motivation of teachers and trainers should be to help and encourage learners, not to demonstrate either how unskilled the learners or how skilled the observer. Giving feedback that makes the teacher feel better or gives the teacher a psychological advantage may serve only to be destructive to the learner.

\section{Change behaviour, NOT personality}

Telling a trainee that there are problems can be very difficult. Giving feedback which separates the person from their professional behaviour can be difficult. Describing someone as a 'loudmouth' or 'talkative' is a comment on an individual's personality, what you think he is. Saying "you seemed to talk quite a lot, the patient tried to interrupt but couldn't quite get into the conversation" is a comment on behavior, what you think he did. ${ }^{6}$ Similarly, saying "You are a illdisciplined student" is a comment on an individual's personality, what you think or communicate he is. Saying "You always arrive late in the duty or in the seminar" is a comment on behaviour. Behavior is easy to alter, personality less so; we are more likely to think we can change what we 'do' than what we 'are'. So while giving feedback, we should focus on behaviour, not on the personality. In fact, feedback may only be given to something that can be checked. There may be little point in reminding someone of a 'shortcoming' that they can not easily remedy. ${ }^{6}$

\section{Descriptive (NOT evaluative or NOT judgemental) language}

The languages used during feedback could be descriptive or evaluative. "I noticed that you avoided eye contact 
in the clinical presentation" is a descriptive language, where as "Your presentation skill was poor" is evaluative one. "Had you thought about asking the patient.......?" is a descriptive language, where as "Your history taking with the patient was totally inadequate" is evaluative one. ${ }^{7}$ Communication skills are neither intrinsically good nor bad, they are just helpful or not helpful in achieving a particular objective in a given situation. We need to address our comments to 'what seemed to work' and 'what didn't seem to work' in relation to getting to where you were trying to go. Descriptive feedback is the process of holding a mirror up for the group: "Here's what I see happened - what do you think?" So instead of "what was done well" and "what could have been done differently", we substitute "what was done" and "what do you think?" ${ }^{6}$ By describing exactly what you see, you almost always produce non-evaluative specific feedback. For descriptive feedback, describe what you saw and reflect back to the student. Descriptive feedback concentrates on what, when, where, and how and avoids comments on why. The SET-GO is one classical method of descriptive feedback used in the Calgary - Cambridge approach to communication skills teaching. ${ }^{6}$ While giving descriptive feedback, facial expressions and body language should be appropriate. The aims of descriptive feedback are to reduce defensiveness, promote an openness of discussion, increase experimentation, aid the presentation of available alternatives and ultimately facilitate change in behavior. By trying to be more descriptive in our language, in our gesture and in our tone of voice, we are attempting to create a non-judgmental climate that encourages learning.

\section{E. Environment}

Feedback can be intimate and personal, people respond in different ways. So environment issues may also be important and so giving feedback in comfortable and safe environment may help. The faculty giving feedback should not appear to be in hurry and may write down their thoughts beforehand to plan.

\section{F. Focused (specific, NOT general)}

Feedback should be focused, that is specific, not generalized. "You used closed questions rather than open questions while taking history of that patient" is specific statement, where as "Your should improve your questioning skills" is general comment. Similarly "You appeared to be rushing with the last three patients" is specific, where as "Time management seems to be a problem" is general. ${ }^{7}$ Suggest specific strategies by which the student might improve their performance. General and vague feedback that does not inform the student is, therefore, unhelpful. Your message will be unclear and may be misinterpreted. If you give balanced, specific and constructive feedback of a piece of work you offer the trainee positive reinforcement of their strengths and an opportunity to learn from their deficiencies. Feedback based on the context is likely to be quite effective.

\section{G. Group check}

If feedback is being given in a group situation, it should be checked with others within the group. Feedback happens within the group, whether we want it or not. The individual group member receives feedback from the group leader and from their peers within the group. This can be a potent way of encouraging change and development. If the feedback process is not planned into the learning event the risk is that misunderstandings, based on non-verbal or unintentional messages occur. The group may become dysfunctional and individuals may become upset. Feedback is, therefore, an essential part of the planning of the group's learning and functions in a variety of ways. As many of the issues may apply to all in the group, feedback in group, particularly using the principles of changing behaviour and being descriptive and focused for the benefits of trainees, may help to develop the culture of giving mutual feedback to each other.

\section{Interpretation check}

During feedback the interpretations should be checked. Feedback should be checked with the recipient, by the recipient and with the others in the group, if it is given in the group situation. Givers of feedback should take responsibility to check out the recipient's response to the feedback. In turn, the recipient should check out whether he has understood the feedback correctly: 'What I think you mean is .......... ${ }^{6}$ Lastly if the feedback has been given in the group situation, it is helpful for both givers and recipients to check out with the rest of the group to see if their impressions are shared by others.

\section{S. Sharing information}

Feedback basically involves sharing information rather than giving advice; this encourages the learner to decide their own course of action. By sharing information, we 
Bhattarai. ABCDEFG IS - The Principle of Constructive Feedback.

leave recipients of feedback free to decide for themselves what the most appropriate course of action is. In contrast, when we give advice, we often tell others what to do and take away their freedom to decide; inadvertently we put them down. There is clearly a fine line in working with learners between sharing and giving advice but we should move away from advice giving as a primary form of giving feedback towards the concept of making offers and suggestions. ${ }^{6}$

\section{Eight Steps of Providing Feedback}

Feedback should be given both informally and formally. ${ }^{7}$ Informal feedback should be frequent and occur in situations where specific behaviours or skills can be discussed in small doses and at the time shortly after. Informal feedback should take place at the time of the event or as soon after while the events are still fresh in the mind. On a day-today basis informal feedback should be given in the context of work activities, whilst observations over a period of time or trends in practice should be shared at a more formal feedback session. The instructor should regard all clinical meetings as opportunities for feedback and should not wait until the end of a rotation. Do it now. Don't put it off. There are steps of providing feedback, which can be adapted to a variety of educational situations. ${ }^{1,6,7}$ To make it easier to remember, the author has described them below in a step-by-step manner.

\section{Ensure prior information}

In formal feedback, ensure trainees are informed they are going to receive feedback. Stress this is for your benefit as well as their own.

\section{Collect data}

Prepare yourself for the meeting by finding the facts. Wherever possible, base the feedback on first hand information i.e. direct observation by yourself, patient notes. ${ }^{7}$ When this is not possible use the relevant data from other people. Try to get specific examples from them to back up their comments. The trainee will not believe the feedback if they feel you have inadequate knowledge of their performance. Make sure you get both sides of the story first. Making notes prior to the meeting helps in planning the feedback.

\section{Make appropriate meeting arrangement}

Demonstrate respect for the trainee e.g. through nonthreatening seating. In a formal feedback session pay attention to the seating arrangements to help ensure the atmosphere is relaxed. Ensure privacy, ensuring mobile phones of both of you are off.

\section{Begin by encouraging self assessment by the trainee} Encourage trainees to self-assess themselves at the beginning of the session eg "How has the period in this unit gone so far - anything in particular you feel was tricky?"7 Encourage the trainee to self assess their performance prior to giving feedback.

\section{Highlight areas where the trainee is doing well}

Reinforce good practice with specific examples. Positive feedback may make students feel good and pleased with the work they have done; this can boost morale and provide positive reinforcement. It reduces defensiveness and promotes openness for discussion. This will inspire confidence and make the trainee more receptive to subsequent negatives.

\section{Give feedback}

Give feedback based on the "ABCDEFG IS", the principles of feedback discussed above. Outline areas which need improvement using descriptive and not evaluative language. Data based on specific actions more appropriately make the point. Identify, analyse and explore potential solutions for any deficits in practice.

\section{Handle reaction maintaining the dignity}

Handle reaction being prepared. Maintain dignity. Making apparently intrusive comments can be awkward, and trainees respond in different ways: aggressively, defensively, or tearfully. Giving your trainee space to express their feelings is important; do not interrupt or confront them. The trainee is attempting to hold on to their dignity; apparently at your expense. ${ }^{1}$ Trainee should be allowed to calm down and then their issues should be addressed. When they are in control of themselves, and are quiet, emphasize the positive, making it clear that the situation can be resolved. Even a feedback session which unintentionally elicits an emotional reaction can be extremely valuable and trainees invariably express appreciation for well-intentioned efforts to help.

\section{Plan actions}

The assessor should ask the trainee for his response to the points raised. Check interpretation of feedback. Make sure the trainee agrees or disagrees with the 
feedback given. Any disagreement by the trainees must be discussed and clarified. The two should discuss plans for the trainee to modify his performance in the future. If the trainee can not come up with potential solutions to negative feedback, make some appropriate suggestions yourself. For planning improvement sixstep problem-solving model is quite useful. The sixstep problem-solving model is a tool used to get agreement between two individuals to solve problems, agreeing goals, aims and objectives. It is particularly useful in trying to agree when things have gone wrong and where the difficulty really lies. The six step problem solving model involves: ${ }^{1}$
i. Problem presented
ii. Problem discussed
iii. Problem agreed
iv. Solution proposed
v. Solution discussed
vi. Solution agreed

The trainee does not usually present with a solution, but more commonly presents with a problem. It is no use trying to solve a problem, if the problem has not been discussed and agreed first. However in some cases it is quite difficult to get the trainee in difficulty to acknowledge that there is a problem in the first place, and then of course it is even more difficult to propose and agree solutions.

\section{Feedback benefits the provider as well}

Giving feedback is communication and in most circumstances communication is a two way process; each person contributes, and therefore each gains something out of the interaction. Feedback benefits both participants. The feedback increases the observation skills of the feedback provider. It also stimulates them to further go in details about the issues related to feedback and to look at the patient management carried out by the residents more objectively. Once we could spell out the problems using descriptive language, discussing and agreeing on the solutions would be relatively easier. The experience of providing feedback increases the vision of looking at things and helps to change the attitude appropriately. It gives insight and stimulates reflection. It also brings the two participants closer. It increases the humane feelings. Medical professionals who make a habit of regularly giving constructive feedback often find it to be one of the most rewarding aspects of their clinical duties.

\section{Encourage Self and Mutual Feedback}

Feedback should be solicited rather than imposed. Feedback is most usefully heard when the recipient has actively sought feedback and has asked for help with specific questions. Encourage trainees to seek feedback themselves from others. Get them to ask people, "How could I have better handled that situation?" As professionals we are also responsible for ensuring we receive feedback upon our own performance. Multisource feedback, also known as $360^{\circ}$ feedback, is a useful and very powerful instrument. ${ }^{1,8-10}$ Colleagues, co-workers, patients, trainees and the individual all provide data which can be amalgamated to provide an overview of aspects of performance, for example, on the seven roles or competencies: medical expert-clinical decision maker, communicator, health advocate, manager, professional, collaborator, and scientistscholar. Multisource feedback, using questionnaire data from patients, medical colleagues, and coworkers, is gaining acceptance and credibility as a means of providing doctors with quality improvement data as part of an overall strategy of maintaining competence. . $^{8,10}$

\section{Feedback, Communication, and Reflection}

The importance of communication in health care setting has been increasingly realized. Miscommunication affects proper patient management and may even lead to medico-legal complaints. Similarly, the ability to reflect on one's practice (performance) is critical to lifelong, self-directed learning. Reflection involves communication with self with relevant feedback to self. Reflecting upon the problem after it was solved (reflection-on-action) and thinking while solving problems (reflection-in-action) are necessary for professionals to gain new insights into their practice, and to develop a life long learning agenda that is truly individually relevant. Both communication and reflection also share many of the principles, particularly change behaviour, descriptive language, interpretation check and sharing information, and the steps, particularly collect data, highlight positive areas, handle reaction maintaining the dignity and plan actions, of constructive feedback. Thus, giving regular feedback helps to improve communication and reflection. The relation between feedback, communication and reflection is depicted by the accompanying diagram. 


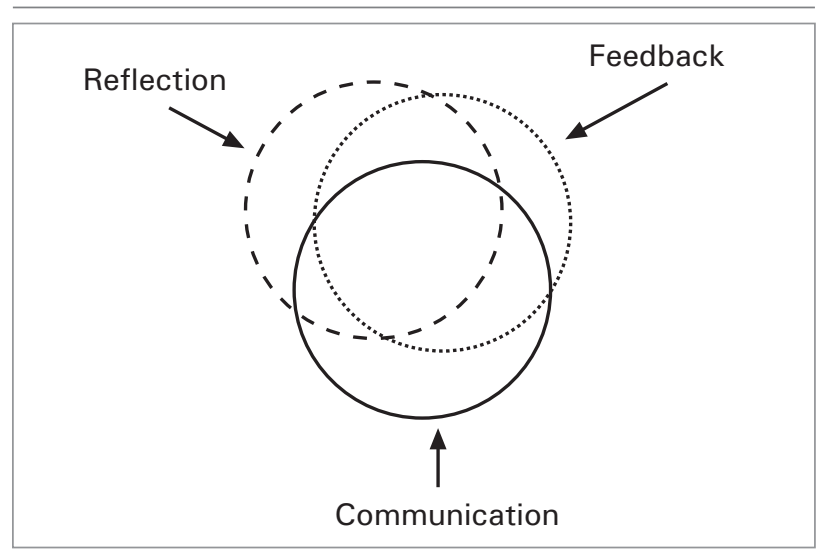

\section{REFERENCES}

1. McCormack A, Law S, Davis M. A. 11 Giving Feedback Theme Assessment. Dundee: Centre for Medical Education 2005.

2. Bruckner H, Altkorn D, Cook S, Quinn MT, McNabb WL. Giving effective feedback to medical students: a workshop for faculty and house staff. Medical Teacher 1999;21:161-165.

3. Gil DH, Heins M, Jones PB. Perception of medical school faculty members and students on clinical clerkship feedback. Journal of Medical Education 1984;59:856-864.

4. Pendleton EA. The consultation; an approach to teaching and learning. Oxford: Oxford University Press 1984.

5. Lempp H, Seale $\mathrm{C}$. The hidden curriculum in undergraduate medical education: qualitative study of medical student's perceptions of teaching. BMJ 2004; 329:7469.

\section{CONCLUSION}

Feedback is part of the learning cycle and is an integral part of any learning experience. It is absolutely critical to get insight into what one did and how one performed and to recognize the discrepancy between the intended and actual behaviour. In this way, every experience could become the learning one, with resultant suitable impetus for change. The concepts of principles and steps of the feedback are discussed here to help achieve such change in the appropriate direction.

6. Silverman J, Draper J, Kurtz SM. The Calgary-Cambridge approach to communication skills teaching II - The SET-GO method of descriptive feedback. Education for General Practice 1997;8:16-23.

7. Hesketh EA, Laidlaw JM. Developing the teaching instinct, 1: Feedback. Medical Teacher 2002;24 (3):245-248.

8. Violato C, Lockyer J, Fidler H. Multi-source feedback: a method of assessing surgical practice. BMJ 2003;326:546-548.

9. Southgate L, Hays RB, Norcini J, Mulholland H, Ayers B, Woolliscroft J, et al. Setting performance standards for medical practice: a theoretical framework. Med Educ 2001;35:478-81.

10. Violato C, Marini A, Toews J, Lockyer J, Fidler H, Toews J. Using peers, consulting physicians, patients, co-workers and self to assess physicians. Acad Med 1997;70:57-63. 